\title{
2016 Kamil Duszenko Prize Goes to Kate Juschenko
}

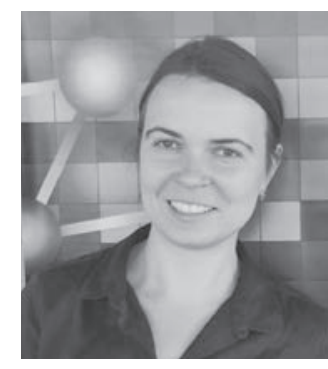

Kate Juschenko of Northwestern University has been named the recipient of the 2016 Kamil Duszenko Award, awarded for outstanding research in Geometric Group Theory.

Kate Juschenko got her $\mathrm{PhD}$ in 2011 at Texas A\& M. Her advisor was Gilles Pisier. She held positions at EPFL, Vanderbilt University and CNRS. She has been at Northwestern University since 2013.

Kate Juschenko started her mathematical education and work in Ukraine. She was then interested in operator algebras, and participated in exploring the notion and providing examples of "-wild $C^{*}$-algebras. She was also engaged in investigating multi-dimensional Schur multilpiers for $C^{*}$-algebras.

Her work connected to geometric group theory is concentrated on the notion of amenability. She was able (with various coauthors) to deeply explore it for groups acting on the Cantor set. To a homeomorphism $T$ of the Cantor set one associates the topological full group [[T]] consisting of homeomorphism piecewise given by powers of $T$. Matui showed that for suitably chosen $T$ the commutator subgroup of $[[T]]$ is finitely generated and simple. Juschenko and Monod proved that these groups are also amenable, thus providing the first examples of finitely generated amenable simple groups. Later, with Nekrashevych and de la Salle she extended this result and devised a new method of proving amenability, settling some open cases and unifying many known ones. Moreover, Juschenko found a powerful method to demonstrate that many groups acting on trees are not elementary amenable.

The Kamil Duszenko Award has been set up in memory of an outstanding young mathematician who died in 2014 of acute lymphoblastic leukaemia at the age of 28 . The webpage with more information is

http://kamil.math.uni.wroc.pl/en/mathematics/about/ 